\title{
Survival Outcomes for Yttrium-90 Transarterial Radioembolization With and Without Sorafenib for Unresectable Hepatocellular Carcinoma Patients
}

This article was published in the following Dove Press journal: Journal of Hepatocellular Carcinoma

Ajalaya Teyateeti, (D) ${ }^{1,2}$ Armeen Mahvash, ${ }^{3}$ James P Long, Mohamed E Abdelsalam, ${ }^{3}$ Rony Avritscher, ${ }^{3}$ Beth Chasen, Ahmed O Kaseb, ${ }^{5}$ Joshua D Kuban, ${ }^{3}$ Ravi Murthy, ${ }^{3}$ Bruno C Odisio, ${ }^{3}$ Achiraya Teyateeti, $\mathbb{D I}^{6}$ Homer A Macapinlac,' S Cheenu Kappadath (iD) ${ }^{7}$

'Department of Nuclear Medicine, Division of Diagnostic Imaging, University of Texas MD Anderson Cancer Center, Houston, TX, USA; '2Division of Nuclear Medicine, Department of Radiology, Faculty of Medicine Siriraj Hospital, Mahidol University, Bangkok, Thailand; ${ }^{3}$ Department of Interventional Radiology, Division of Diagnostic Imaging, University of Texas MD Anderson Cancer Center, Houston, TX, USA; ${ }^{4}$ Department of Biostatistics, University of Texas MD Anderson Cancer Center, Houston, TX, USA; ${ }^{5}$ Department of Gastrointestinal Medical Oncology, Division of Cancer Medicine, University of Texas MD Anderson Cancer Center, Houston, TX, USA; ${ }^{6}$ Division of Radiation Oncology, Department of Radiology, Faculty of Medicine Siriraj Hospital, Mahidol University, Bangkok, Thailand; ${ }^{7}$ Department of Imaging Physics, Division of Diagnostic Imaging, University of Texas MD Anderson Cancer Center, Houston, TX, USA

Correspondence: S Cheenu Kappadath Department of Imaging Physics, Division of Diagnostic Imaging, University of Texas MD Anderson Cancer Center, II 155 Pressler Street - Unit 1352, Houston, TX 77030, USA

Tel +I 7137452835

Email skappadath@mdanderson.org
Purpose: To assess the overall survival (OS) and progression-free survival (PFS) of unresectable hepatocellular carcinoma (HCC) patients undergoing yttrium-90 glass-microsphere transarterial radioembolization (TARE) with and without concurrent sorafenib.

Methods: OS and PFS were analyzed in 55 patients with an intrahepatic tumor (IHT) $\leq 50 \%$ without advanced or aggressive disease features (ADFs), which was referred to presence of infiltrative/ill-defined HCC, macrovascular invasion, or extrahepatic disease treated with only TARE (TARE alone) and in 74 patients with IHT $\leq 50 \%$ with ADFs or IHT $>50 \%$ treated with TARE and sorafenib (TARE_sorafenib). Prognostic factors for OS and PFS were identified using univariate and multivariate analyses.

Results: Median OS and PFS of TARE_alone patients were 21.6 (95\% CI 6.1-37.1) and 9.1 (95\% CI 5.2-13.0) months, respectively, and for TARE_sorafenib patients $12.4(95 \% \mathrm{CI}$ 9.1-15.6) and 5.1 (95\% CI 2.6-7.5) months, respectively. Better OS was associated with serum AFP $<400$ (HR 0.27, $p=0.02$ ) in TARE_alone, and IHT $\leq 50 \%$ (HR $0.39, p=0.004)$ and AFP $<400$ (HR 0.5, $p=0.027$ ) in TARE_sorafenib. Unilobar involvement (HR 0.43, $p=0.029$ ) and AFP $<400 \mathrm{ng} / \mathrm{mL}$ (HR 0.52, $p=0.015)$ correlated with better PFS in TARE_alone and TARE_sorafenib, respectively. Adverse events (AEs) were more frequent in TARE_sorafenib than TARE_alone ( 92.4 vs $80.3 \%$ ), but only $9.3 \%$ were grade 3 or higher AEs.

Conclusion: TARE_alone provided the most prominent survival benefit in IHT $\leq 50 \%-$ without ADF patients who had unilobar HCC and serum AFP $<400 \mathrm{ng} / \mathrm{mL}$. TARE and sorafenib yielded the best outcomes in patients with IHT $\leq 50 \%$ and serum AFP $<400 \mathrm{ng} / \mathrm{mL}$, with some additional grade 1-2 AEs compared to TARE only.

Keywords: ${ }^{90} \mathrm{Y}$, selective internal radiation therapy, TheraSphere, prognostic factors, adverse events

\section{Introduction}

Hepatocellular carcinoma (HCC) is currently the sixth-most common cancer and fourth-most common cause of cancer-related death worldwide, according to World Health Organization data. ${ }^{1}$ Potentially curative treatments, namely liver transplantation and surgical resection, are amenable in only $30 \%-40 \%$ of patients. ${ }^{2,3}$ The rest require either locoregional therapies, such as ablation or transarterial chemoembolization (TACE), or systemic therapy, such as sorafenib and regorafenib.,

Transarterial radioembolization (TARE), or selective internal radiotherapy is a form of local therapy that delivers high dosages of yttrium-90 $\left({ }^{90} \mathrm{Y}\right)$-radiolabeled microspheres to tumors. Many studies regarding the efficacy of ${ }^{90} \mathrm{Y}$ TARE (or 
TARE henceforth) have been published over the last decade. ${ }^{6-13}$ The reported overall survival (OS) of unresectable HCC patients undergoing TARE ranges from 7 to 27 months. ${ }^{8-10,12,13}$ This range are exceptionally wide, likely because unresectable HCC includes disparate disease burdens (DBs), ranging from solitary lesions to multifocal or metastatic disease. The markedly short OS of the Barcelona Clinic Liver Cancer (BCLC) stage B with multiple large tumors and BCLC stage $\mathrm{C}$ patients led to the investigation of TARE plus sorafenib. A few studies of this combined treatment have suggested acceptable toxicity, but survival benefits remain questionable. ${ }^{14-16}$ Their varied outcomes probably contributed to a mix of advanced DBs. As a result, appropriate patient selection might be key to improving outcomes of this combined treatment.

The multidisciplinary team at our institution has three major considerations for HCC-treatment assessment: percentage of intrahepatic tumor burden (IHT), presence of aggressive or advanced disease features (ADFs), and general condition of patients. Patients with ADFs or IHT $>50 \%$ tend to receive combined treatment, commonly TARE and sorafenib, while those with contraindications to sorafenib or IHT $\leq 50 \%$ without ADFs usually receive TARE only. ${ }^{17}$ Our institutional treatment schema differs from the conventional BCLC stage-based approaches, and consequently treatment outcomes at our institution need evaluation, as they may differ from previous publications. The objective of this study was to quantitatively establish the practice-based outcomes of our institutional HCC treatment approach: TARE with and without sorafenib. The primary objective was determination of OS, whereas progression-free survival (PFS), identification of prognostic factors for OS and PFS, and treatment-related adverse events (AEs) were the secondary objectives investigated in this study.

\section{Methods}

\section{Patient Selection}

This retrospective study looked at HCC patients who had undergone TARE with ${ }^{90}$ Y-glass microspheres (TheraSphere; BTG Biocompatibles, UK) as part of the standard of care under an institutional review board approved protocol at our institution from November 16, 2010 to October 1, 2018. Inclusion criteria were patients who had radiologically or pathologically confirmed HCC, Child-Pugh class A (score 5-6) or B (score 7), had unresectable disease, as assessed by an institutional multidisciplinary team composed of medical oncologists, surgeons, radiation oncologists, and interventional radiologists, and were candidates for TARE with or with sorafenib. The original cohort consisted of 197 TARE candidates, in which 42 were later excluded, due to lack of adequate follow-up $(n=13)$, potentially excessive radiation to normal tissue (lung dose $>30$ Gy for a single treatment or $>50$ Gy for multiple treatments $[n=10]$ ), uncorrectable extrahepatic flow $(\mathrm{n}=1)$, significant intrahepatic shunt $(\mathrm{n}=1)$, poor tumor perfusion evident from angiography, cone-beam computerized tomography (CT), CT angiography, or ${ }^{99 \mathrm{~m}} \mathrm{Tc}$ macroaggregated albumin (MAA) pretreatment scan $(n=7)$, rapid decline of general condition, or disease progression while waiting for TARE $(n=10)$, yielding 155 eligible patients for the analysis. ${ }^{18}$

\section{Pretreatment Evaluation}

Pretreatment evaluations, including clinical histories and physical examinations from medical records, radiology reports from baseline imaging, and laboratory tests, were reviewed retrospectively for all patients. We determined patients' BCLC stage and Eastern Cooperative Oncology Group (ECOG) performance status. ${ }^{4,19}$ Laboratory workups consisted of $\alpha$-fetoprotein (AFP), liver-function tests, biochemistry, and hematology. Hepatic function was evaluated using Child-Pugh class and albumin-bilirubin (ALBI) grade. ${ }^{20}$ The most recent radiology reports from contrast-enhanced CT or magnetic resonance imaging, preferably obtained within 2 months before TARE, were used to evaluate the number of tumors, lobar involvement, IHT burden, macrovascular invasion (MVI), lymph node metastasis, distant metastasis, cirrhosis, and ascites.

\section{Treatment}

Three major variables were assessed by a multidisciplinary team: IHT ( $\leq 50$ vs $>50 \%$ ), absence or presence of ADFs (defined as infiltrative/ill-defined HCC, MVI, and extrahepatic disease [EHD]), and patient condition (ECOG and underlying diseases). Then, one of the treatment strategies of TARE_alone, TARE_sorafenib, and TARE_alternative was chosen according to team consensus (Table 1). All patients underwent a ${ }^{99 \mathrm{~m}} \mathrm{Tc}$ MAA pretreatment scan with single-photon emission computed tomography (SPECT)/ CT imaging over thoracic and abdominal regions to assess vascular anatomy, simulate ${ }^{90} \mathrm{Y}$-microsphere distribution. Administered ${ }^{90}$ Y-activity was based on the device's package-insert single-compartment medical internal radiationdose dosimetry. The administered activity was deemed 
Table I Stratification of Patients in Terms of Treatment Strategy

\begin{tabular}{|l|l|l|}
\hline & Patients, $\mathbf{n}$ & Characteristics \\
\hline TARE_alone & 55 & $\begin{array}{l}\text { TARE as single treatment for IHT } \\
\leq 50 \% \text { without ADFs }\end{array}$ \\
\hline TARE_sorafenib & 74 & $\begin{array}{l}\text { TARE and sorafenib as combined } \\
\text { treatment for IHT } \leq 50 \% \text { with } \\
\text { ADFs or IHT }>50 \%\end{array}$ \\
\hline TARE_alternative & 26 & $\begin{array}{l}\text { TARE as alternative treatment } \\
\text { for patients who should have } \\
\text { receive TARE_sorafenib, but had } \\
\text { unsuitable conditions or } \\
\text { contraindications to sorafenib }\end{array}$ \\
\hline Total & 155 & \\
\hline
\end{tabular}

decreased if mean lung-absorbed dose was predicted to be $>30$ Gy based on each patient's lung-shunt fraction from ${ }^{99 \mathrm{~m}}$ Tc MAA geometric-mean images. The median (range) of lung-shunt fractions was $6.0 \%(0.8 \%-30.4 \%)$. Eligible patients subsequently underwent TARE, typically within 1 month. ${ }^{90} \mathrm{Y}$ microspheres were administered following the manufacturer's guidelines. ${ }^{21}$ The median (range) of administered activity was $2.6(0.5-8.1) \mathrm{GBq}$. The median (range) of mean absorbed dose to treated liver volume was $110(80-135)$ Gy. Sorafenib was given concurrently or within a 1-month interval, either before or after TARE. Dosage of sorafenib was adjusted by the medical oncologists from 200 to $800 \mathrm{mg}$ per day on the basis of tolerability, and temporary withdrawal was allowed in cases of severe AEs. ${ }^{22}$ Patients with contraindications or side-effect intolerance to sorafenib were able to stop sorafenib and switch to other systemic therapy or receive only TARE.

\section{Post-treatment Evaluation}

Clinical and biochemical follow-up were scheduled at 1 month and 3 months after TARE and every 2-4 months thereafter, and assessed in a procedure-based fashion according to National Cancer Institute's Common Terminology Criteria for Adverse Events (CTCAE; version 5.0). ${ }^{23}$ AEs were defined as any event that occurred after TARE until the patient's death, last follow-up, or change of treatment, whichever came first. Unmeasurable parameters (eg, ascites and fatigue) and preexisting abnormal measurable parameters were considered AEs if the CTCAE grade had changed after the procedure or if they required close followup or treatment. Radioembolization-induced liver disease (REILD) was defined as total serum bilirubin $\geq 3 \mathrm{mg} / \mathrm{dL}$ and ascites in the absence of tumor progression or bile-duct obstruction. ${ }^{24}$ Radiological follow-up was obtained at 2 months after TARE and then every 2-3 months. Tumor response was assessed using Response Evaluation Criteria in Solid Tumors version 1.1. ${ }^{25}$ Evaluation was performed until disease progression, last radiological follow-up, or patient death, whichever came first. The date of disease progression was the date of the first radiological evidence of disease progression that resulted in change in management.

\section{Outcome Assessment and Prognostic Factor Identification}

OS duration was defined as time from the first TARE to death from any cause. Patients who were alive at last follow-up were treated as censored. PFS duration was defined as time from first TARE to disease progression at any site or death from any cause, whichever occurred first. Patients without disease progression or death were censored at the time of the last follow-up with no evidence of disease progression. OS and PFS durations were estimated in the three treatment subgroups (Table 1). Prognostic factors were analyzed only in the major population subgroups: TARE_alone and TARE_sorafenib.

\section{Statistical Analysis}

OS and PFS durations were analyzed using the KaplanMeier method, median values and 95\% CIs calculated, and comparison of survival curves across subgroups done using the log-rank test. A univariate analysis to identify potentially prognostic factors was performed using the Kaplan-Meier method. All factors in the univariate analysis with $p<0.1$ were further analyzed in a multivariate model. To eliminate the crossover effect, certain composite factors in which one or more constituent factors were already included in the multivariate analysis were excluded (eg, excluded BCLC stage, if ECOG was already included in the analysis). A multivariate analysis of prognostic factors was performed using a Cox proportional-hazard model via the HR. Statistical significance on multivariate analysis was $p<0.05$. All statistical analyses were conducted using SPSS version 21.0.

\section{Results}

\section{Patient, Tumor, and Treatment Characteristics}

The median follow-up period of the entire population was 8.8 (range 1.2-76.1) months. Patients' principal 
demographic characteristics and disease presentations stratified by treatments are summarized in Table 2: of the 155 patients, most had BCLC stage $\mathrm{C}(71 \%)$ and presented with multifocal HCC (83.2\%), bilobar involvement (60\%), IHD $\leq 50 \%$ (68.4\%), and AFP $<400 \mathrm{ng} / \mathrm{mL}$ (69.5\%). MVI and EHD were present in $25.8 \%$ and $19.4 \%$ of patients, respectively. There were $48.4 \%$ and $44.5 \%$ of patients undergoing at least one treatment before and after TARE, respectively (Table 3). Patients undergoing one, two, and three sessions of TARE were $89 \%$, $9 \%$, and $2 \%$, respectively. Median intervals between first and second TARE and between second and third TARE were 8.9 (2.1-49.6) and $9.4(2.5-19.8)$ months, respectively. The largest treatment subgroups were TARE_alone (35.5\%) and TARE_sorafenib (47.7\%). Of 74 patients in the TARE_sorafenib subgroup, 43 (58.1\%) received suboptimal doses of sorafenib, due to side-effect intolerance. Sorafenib was given prior to TARE in 67 patients $(90.5 \%)$. The median time from initiation of sorafenib to TARE was 1.5 months, with a range of 74.5 months before to 0.6 months after TARE.

\section{Survival Analysis}

Median OS and PFS of the overall population were 14.4 (95\% CI 10.6-18.2) and 5.1 (95\% CI 3.3-6.8) months, respectively. OS rates at 1,2 , and 3 years were $54.1 \%$, $36.1 \%$, and $29 \%$, respectively, and PFS rates at 1 and 2 years $25.6 \%$ and $9.7 \%$, respectively. OS and PFS durations of the subgroups are shown in Table 4. Patients with IHT $\leq 50 \%$ without ADFs receiving TARE_alone had the longest median OS of 21.6 (95\% CI 6.1-37.1) months and PFS of 9.1 (95\% CI 5.2-13.0) months. OS rates of the TARE_alone population at 1, 2, and 3 years were $65.3 \%$, $49.8 \%$, and $35.4 \%$, respectively. Patients with IHT $\leq 50 \%$ with ADFs or IHT $>50 \%$ receiving TARE_sorafenib showed better OS (12.4 vs 7.3 months) and PFS (5.1 vs 2.8 months) than TARE_alternative treatments. OS rates of the TARE_sorafenib population at 1,2, and 3 years were $53.2 \%, 34 \%$, and $28 \%$, respectively, and those of the TARE_alternative population at 1 and 2 years $31.4 \%$ and $15.7 \%$, respectively.

\section{Prognostic Factors}

Univariate analysis results of OS and PFS for the TARE_alone and TARE_sorafenib subgroups are shown in Table 5. Statistically significant differences in OS and PFS for TARE_alone patients were observed when stratified with AFP (OS 35.2 vs 5.0 months, $p<0.001$; PFS 10.2 vs 2.2 months, $p=0.005$ ), lobar involvement (OS 27.4 vs 10.2 months, $p=0.025$ and PFS 12.3 vs 4.7 months, $p=0.038$ ) and ECOG (PFS 12.2 vs 4.7 months, $p=0.038$ ). The sole variables affecting the TARE_sorafenib subgroup were IHT (OS 21.6 vs 11.3 months, $p=0.024$ ) and sex (PFS 6.7 vs 2.8 months, $p=0.002$ ), respectively.

Multivariate analysis results for OS and PFS are shown in Table 6. Prognostic factors for OS were AFP (HR 0.27, 95\% CI 0.09-0.81; $p=0.02$ ) for the TARE_alone subgroup (Figure 1A) and AFP (HR 0.5, 95\% CI 0.27-0.93; $p=0.027$ ) and IHT (HR 0.39, 95\% CI 0.2-0.74; $p=0.004$ ) for the TARE_sorafenib subgroup (Figure 1B). BCLC stage was excluded from the multivariate analysis of PFS for the TARE_alone subgroup, as ECOG, one of its constituent factors, was already included. The prognostic factor for PFS in the TARE_alone subgroup was lobar involvement (HR 0.43 , 95\% CI $0.20-0.92 ; p=0.029$; Figure 1C), and for the TARE_sorafenib subgroup prognostic factors were AFP (HR $0.52,95 \%$ CI $0.30-0.88$; $p=0.015$ ) and sex (HR $0.46,95 \%$ CI $0.24-0.87 ; p=0.018$; Figure 1D).

\section{AEs}

There were 549 AEs identified in 149 of 175 procedures (85.1\%). Number of AEs and pertinent AEs stratified by treatment are displayed in Table 7. AEs were most frequently seen in the TARE_sorafenib subgroup (92.4\%), followed by the TARE_alone subgroup (80.3\%). Number of AEs per procedure was highest in the TARE_sorafenib (median three, range 0-11) and TARE_alternative (median 3, range 0-8) subgroups. Dosages of sorafenib were reduced in 44 of 79 TARE_sorafenib procedures (55.7\%). The five most common AEs in all treatment subgroups were fatigue, nausea/vomiting, abdominal discomfort, hyperbilirubinemia, and thrombocytopenia. Dermatological symptoms (26.6\%) and diarrhea (19.0\%) were more often observed in the TARE_sorafenib subgroup than the other subgroups (Table 8).

Grade 1-2 AEs accounted for $90.7 \%$ of total AEs, while grade 3-4 AEs accounted for 9.3\%. No patients experienced grade 5 AEs or serious consequences, such as radiation pneumonitis or REILD. The number of grade 3-4 AEs per procedure was slightly higher in the TARE_alone (median two, range 1-6) than the TARE_sorafenib (median one, range 1-4) and TARE_alternative (median one, range 1-2) subgroups. Probable treatment-related complications included grade 
Table 2 Principal Characteristics of Patients and Disease Presentations

\begin{tabular}{|c|c|c|c|c|}
\hline & $\begin{array}{l}\text { All Patients } \\
(n=155)\end{array}$ & $\begin{array}{l}\text { TARE_alone } \\
(n=55)\end{array}$ & $\begin{array}{l}\text { TARE_sorafenib } \\
(n=74)\end{array}$ & $\begin{array}{l}\text { TARE_alternative } \\
(n=26)\end{array}$ \\
\hline Median age, years (range) & $65(15-85)$ & $70(29-84)$ & $63(27-84)$ & $64.5(17-85)$ \\
\hline Patients aged $<65$ years, $n(\%)$ & $71(45.8)$ & $15(27.3)$ & $43(58.1)$ & $13(50)$ \\
\hline Patients aged $\geq 65$ years, $n(\%)$ & $84(54.2)$ & $40(72.7)$ & $31(41.9)$ & $13(50)$ \\
\hline \multicolumn{5}{|l|}{ Sex, n (\%) } \\
\hline Male & $117(75.5)$ & $42(76.4)$ & $58(78.4)$ & $17(65.4)$ \\
\hline Female & $38(24.5)$ & $13(23.6)$ & $16(21.6)$ & $9(34.6)$ \\
\hline \multicolumn{5}{|l|}{ Ethnicity, n (\%) } \\
\hline Non-Hispanic white & $109(70.3)$ & $46(83.6)$ & $46(62.2)$ & $17(65.4)$ \\
\hline Hispanic & $16(10.3)$ & $5(9.1)$ & $9(12.2)$ & $2(7.7)$ \\
\hline Asian & $16(10.3)$ & $\mathrm{I}(\mathrm{I} .8)$ & $10(13.5)$ & $5(19.2)$ \\
\hline Black & $14(9.0)$ & $3(5.5)$ & $9(12.2)$ & $2(7.7)$ \\
\hline \multicolumn{5}{|l|}{ Etiology, n (\%) } \\
\hline Hepatitis B & $17(11.0)$ & $3(5.5)$ & $13(17.6)$ & I (3.8) \\
\hline Hepatitis C & $52(33.5)$ & $22(40)$ & $20(27)$ & $10(38.5)$ \\
\hline Metabolic & $36(23.2)$ & $17(30.9)$ & $14(18.9)$ & $5(19.2)$ \\
\hline Alcoholic & II (7.1) & $4(7.3)$ & $5(6.8)$ & $2(7.7)$ \\
\hline Combined & $6(3.9)$ & 0 & $6(8.1)$ & 0 \\
\hline Unknown & $33(21.3)$ & $9(16.4)$ & $16(21.6)$ & $8(30.8)$ \\
\hline \multicolumn{5}{|l|}{ Tumor differentiation, $\mathbf{n}(\%)$} \\
\hline Well & $41(26.5)$ & $13(23.6)$ & $19(25.7)$ & $9(34.6)$ \\
\hline Moderately & $50(32.3)$ & $17(30.9)$ & $25(33.8)$ & $8(30.8)$ \\
\hline Poorly & $17(11)$ & $3(5.5)$ & $10(13.5)$ & $4(15.4)$ \\
\hline Unknown & $10(6.5)$ & $4(7.3)$ & $5(6.8)$ & I (3.8) \\
\hline Fibrolamellar & $3(1.9)$ & I (I.8) & I (I.4) & $\mathrm{I}(3.8)$ \\
\hline No biopsy & $34(21.9)$ & $17(30.9)$ & $14(18.9)$ & $3(11.5)$ \\
\hline \multicolumn{5}{|l|}{ ECOG, $n(\%)$} \\
\hline 0 & $75(48.4)$ & $27(49.1)$ & $38(51.4)$ & $10(38.5)$ \\
\hline 1 & $74(47.7)$ & $25(45.5)$ & $34(45.9)$ & $15(57.7)$ \\
\hline 2 & $5(3.2)$ & $2(3.6)$ & $2(2.7)$ & I (3.8) \\
\hline 3 & $\mathrm{I}(0.6)$ & $\mathrm{I}(\mathrm{I} .8)$ & 0 & 0 \\
\hline \multicolumn{5}{|l|}{ BCLC, n (\%) } \\
\hline A & $5(3.2)$ & $5(9.1)$ & 0 & 0 \\
\hline B & $39(25.2)$ & $22(40)$ & $16(21.6)$ & I (3.8) \\
\hline $\mathrm{C}$ & $110(71.0)$ & $27(49.1)$ & $58(78.4)$ & $25(96.2)$ \\
\hline $\mathrm{D}$ & I (0.6) & I (I.8) & 0 & 0 \\
\hline \multicolumn{5}{|l|}{ Cirrhosis, n (\%) } \\
\hline Absence & $49(31.6)$ & $13(23.6)$ & $27(36.5)$ & $9(34.6)$ \\
\hline Presence & $106(68.4)$ & $42(76.4)$ & $47(63.5)$ & $17(65.4)$ \\
\hline \multicolumn{5}{|l|}{ Ascites, n (\%) } \\
\hline Absence & $|4|(9 \mid)$ & $49(89.1)$ & $69(93.2)$ & $23(88.5)$ \\
\hline Presence & $14(9)$ & $6(10.9)$ & $5(6.8)$ & $3(11.5)$ \\
\hline \multicolumn{5}{|l|}{ Vascular invasion, $\mathbf{n}(\%)$} \\
\hline Absence & $115(74.2)$ & $55(100)$ & $48(64.9)$ & $12(46.2)$ \\
\hline Portal vein & $36(23.2)$ & 0 & $22(29.7)$ & $14(53.8)$ \\
\hline Hepatic vein & $3(1.9)$ & 0 & $3(4.1)$ & 0 \\
\hline Inferior vena cava & $\mathrm{I}(0.6)$ & 0 & $\mathrm{I}(\mathrm{l} .4)$ & 0 \\
\hline
\end{tabular}


Table 2 (Continued).

\begin{tabular}{|c|c|c|c|c|}
\hline & $\begin{array}{l}\text { All Patients } \\
(n=\mid 55)\end{array}$ & $\begin{array}{l}\text { TARE_alone } \\
(n=55)\end{array}$ & $\begin{array}{l}\text { TARE_sorafenib } \\
(n=74)\end{array}$ & $\begin{array}{l}\text { TARE_alternative } \\
(n=26)\end{array}$ \\
\hline \multicolumn{5}{|l|}{ Lymph node metastasis, n (\%) } \\
\hline Absence & $137(88.4)$ & $55(100)$ & $63(85.1)$ & $19(73.1)$ \\
\hline Presence & $18(11.6)$ & 0 & II (14.9) & $7(26.9)$ \\
\hline \multicolumn{5}{|l|}{ Distant metastasis, n (\%) } \\
\hline Absence & $135(87.1)$ & $55(100)$ & $61(82.4)$ & $19(73.1)$ \\
\hline Presence & $20(12.9)$ & 0 & $13(17.6)$ & $7(26.9)$ \\
\hline \multicolumn{5}{|l|}{ Extrahepatic disease, ${ }^{\mathrm{a}}$ n (\%) } \\
\hline Absence & $125(80.6)$ & $55(100)$ & $55(74.3)$ & I5 (57.7) \\
\hline Presence & $30(19.4)$ & 0 & $19(25.7)$ & II (42.3) \\
\hline \multicolumn{5}{|l|}{ Number of tumors, $\mathbf{n}(\%)$} \\
\hline Single & $26(16.8)$ & $10(18.2)$ & $9(12.2)$ & $7(26.9)$ \\
\hline Multiple & $129(83.2)$ & $45(81.8)$ & $65(87.8)$ & $19(73.1)$ \\
\hline \multicolumn{5}{|l|}{ Lobar involvement, $\mathbf{n}(\%)$} \\
\hline Unilobar & $62(40)$ & $32(58.2)$ & $20(27)$ & $10(38.5)$ \\
\hline Bilobar & $93(60)$ & $23(41.8)$ & $54(73)$ & $16(6 \mid .5)$ \\
\hline \multicolumn{5}{|l|}{ Intrahepatic tumor, n (\%) } \\
\hline$\leq 50 \%$ & $106(68.4)$ & $55(100)$ & $34(45.9)$ & $17(65.4)$ \\
\hline$>50 \%$ & $49(31.6)$ & 0 & $40(54.1)$ & $9(34.6)$ \\
\hline AFP (ng/mL) & $68.0(1.5-656,373.0)$ & $18.2(1.9-109,307.7)$ & $102.9(1.5-656,373.0)$ & $240.2(2.2-44,508.4)$ \\
\hline$<400$ & $107(69.5)$ & $45(81.8)$ & $47(63.5)$ & $15(60)$ \\
\hline$\geq 400$ & $47(30.5)$ & $10(18.2)$ & $27(36.5)$ & $10(40)$ \\
\hline \multicolumn{5}{|l|}{ Albumin-bilirubin grade, n (\%) } \\
\hline 1 & $82(52.9)$ & $28(50.9)$ & $4 \mathrm{I}(55.4)$ & $13(50)$ \\
\hline 2 & $73(47.1)$ & $27(49.1)$ & $33(44.6)$ & $13(50)$ \\
\hline \multicolumn{5}{|l|}{ Child-Pugh class, n (\%) } \\
\hline A & 144 (92.9) & $50(90.9)$ & 71 (95.9) & $23(88.5)$ \\
\hline B & $11(7.1)$ & $5(9.1)$ & $3(4.1)$ & $3(11.5)$ \\
\hline
\end{tabular}

Notes: ${ }^{a}$ Included lymph node and distant metastasis; ${ }^{b}$ AFP unavailable in one TARE_alternative patient.

3 acute pancreatitis in one TARE_alone patient that developed 1 day after TARE, and grade 1 radiation-induced dermatitis in 1 TARE_sorafenib patient observed immediately after TARE.

\section{Discussion}

While most survival analyses of HCC patients undergoing TARE have been based on BCLC staging, in this work our patient-treatment strategy was based on IHT ( $\leq 50 \mathrm{vs}>50 \%)$, ADFs (presence or absence of ill-defined or infiltrative HCC, MVI, or EHD), and performance status. We also allowed for combined treatment with sorafenib in relatively high DB (IHT $>50 \%$ or present ADFs) and other treatment options; therefore, clinical outcomes were unknown.
The overall median OS of 14.4 months and median PFS of 5.1 months in our population was found to be similar to other studies. $^{8-10,12,13}$ Interestingly, TARE_alone patients had the longest OS (21.6 months) and PFS (9.1 months), despite receiving monotherapy, inferring that TARE_alone is a promising treatment for patients with IHT $\leq 50 \%$ without ADFs. Furthermore, patients who should have received TARE_sorafenib (IHT $>50 \%$ or presence of ADFs), but had contraindications to sorafenib or could not tolerate sorafenib (TARE_alternative) experienced the shortest OS (7.3 months) and PFS (2.8 months). A novel finding was observed in the TARE_sorafenib subgroup whereby OS of patients with IHT $\leq 50 \%$ was almost twice as long as those with IHT $>50 \%$ ( 21.6 vs 11.3 months, $p=0.024$ ). Additionally, 
Table 3 Treatment Characteristics

\begin{tabular}{|c|c|c|c|c|}
\hline & $\begin{array}{l}\text { All Patients } \\
(n=\mid 55)\end{array}$ & $\begin{array}{l}\text { TARE_alone } \\
(n=55)\end{array}$ & $\begin{array}{l}\text { TARE_sorafenib } \\
(n=74)\end{array}$ & $\begin{array}{l}\text { TARE_alternative } \\
(n=26)\end{array}$ \\
\hline \multicolumn{5}{|l|}{ Treatment before TARE, $n$ (\%) } \\
\hline None & $80(51.6)$ & $30(54.5)$ & $44(59.5)$ & $6(23.1)$ \\
\hline Local $^{\mathrm{a}}$ & $50(32.3)$ & $17(30.9)$ & $26(35.1)$ & $7(26.9)$ \\
\hline Systemic ${ }^{b}$ & $25(16.1)$ & $8(14.5)$ & $4(5.4)$ & $13(50)$ \\
\hline \multicolumn{5}{|l|}{ TARE $^{\mathrm{c}}$} \\
\hline Median LSF, \% (range) & $6.0(1.1-30.4)$ & $4.6(1.1-26.4)$ & $6.3(1.2-30.4)$ & $6.6(2.0-20.8)$ \\
\hline Median lung dose, Gy (range) & $8.5(0.5-49.0)$ & $4.7(0.5-29.2)$ & II.5 (0.6-49.0) & $12.7(2.0-29.2)$ \\
\hline Median mean absorbed dose to treated liver volume, Gy (range) & $110(80-135)$ & $110(80-135)$ & $110(80-135)$ & $110(80-135)$ \\
\hline Median interval between ${ }^{99 \mathrm{~m}} \mathrm{Tc}$ MAA and TARE, days (range) & $20(0-78)$ & $21(0-78)$ & $18(0-44)$ & $21(10-34)$ \\
\hline Median administered activity $\left(\mathrm{GBq}^{\mathrm{d}}\right)$, range & $2.9(0.5-8.1)$ & $1.9(0.5-5.8)$ & $3.0(0.6-8.1)$ & $2.6(0.8-6.3)$ \\
\hline \multicolumn{5}{|l|}{ TARE procedures, $\mathrm{n}(\%)$} \\
\hline 1 & $138(89)$ & $47(85.5)$ & $66(89.2)$ & $25(96.2)$ \\
\hline 2 & $14(9)$ & $7(12.7)$ & $7(9.5)$ & 0 \\
\hline 3 & $3(1.9)$ & I ( $(1.8)$ & $\mathrm{I}(\mathrm{l} .4)$ & I (3.8) \\
\hline \multicolumn{5}{|l|}{ Approach } \\
\hline Whole liver & $40(23.7)$ & II (20) & $21(28.4)$ & $5(19.2)$ \\
\hline Lobar & $124(73.4)$ & $4 \mid(74.5)$ & $51(68.9)$ & $21(80.8)$ \\
\hline Segmental & $5(3.0)$ & $3(5.5)$ & $2(2.7)$ & 0 \\
\hline \multicolumn{5}{|l|}{ Treatment after TARE, n (\%) } \\
\hline None $e^{e}$ & $50(32.3)$ & $25(45.5)$ & $21(28.4)$ & $4(15.4)$ \\
\hline Best supportive care & $36(23.2)$ & $8(14.5)$ & $15(20.3)$ & $13(50)$ \\
\hline Local & $22(14.2)$ & $12(2 \mid .8)$ & $8(10.8)$ & $2(7.7)$ \\
\hline Systemic & $47(30.3)$ & $10(18.2)$ & $30(40.5)$ & 7 (26.9) \\
\hline
\end{tabular}

Notes: ${ }^{a}$ Included surgical resection, transarterial embolization (TAE), transarterial chemoembolization (TACE), TARE, radiofrequency ablation (RFA), and microwave ablation (MWA). 'Included chemotherapy, targeted therapy and immunotherapy. ${ }^{\mathrm{C} F i r s t}$ TARE only. ${ }^{\mathrm{d}}$ Gigabecquerel. ${ }^{\mathrm{e}}$ No treatment because of no progression.

Table 4 OS and PFS Stratified by Treatment Strategy

\begin{tabular}{|c|c|c|c|c|c|}
\hline & \multirow{2}{*}{$\begin{array}{l}\text { Total } \\
\text { Patients }\end{array}$} & \multicolumn{2}{|l|}{ OS } & \multicolumn{2}{|l|}{ PFS } \\
\hline & & $\begin{array}{l}\text { Censored } \\
\text { Patients }\end{array}$ & $\begin{array}{l}\text { Median }(95 \% \mathrm{Cl}) \text {, } \\
\text { Months }\end{array}$ & $\begin{array}{l}\text { Censored } \\
\text { Patients }\end{array}$ & $\begin{array}{l}\text { Median }(95 \% \mathrm{CI}) \text {, } \\
\text { Months }\end{array}$ \\
\hline Entire population & 155 & 76 & $14.4(11.0-17.9)$ & 32 & $5.1(3.3-6.8)$ \\
\hline \multicolumn{6}{|l|}{ Treatment } \\
\hline TARE_alone & 55 & 33 & $21.6(6 . I-37 . I)$ & 20 & $9.1(5.2-13.0)$ \\
\hline TARE_sorafenib & 74 & 25 & $12.4(9.1-15.6)$ & 7 & $5.1(2.6-7.5)$ \\
\hline TARE_alternative ${ }^{a}$ & 26 & 11 & $7.3(4.7-9.8)$ & 2 & $2.8(1.8-3.8)$ \\
\hline
\end{tabular}

Notes: ${ }^{a}$ Did not receive sorafenib because of side-effect intolerance $(n=12)$, no or delayed follow-up with the medical oncologist $(n=4)$, contraindications to sorafenib $(n=8)$ and other recent systemic treatment $(n=2)$.

TARE_sorafenib patients with IHT $\leq 50 \%$ with ADFs achieve OS of 21.6 months, similar to TARE_alone patients. These findings strongly suggest a survival benefit from TARE_sorafenib in patients with IHT $\leq 50 \%$ and presence of ADFs.

Besides the well-known effect of devascularization, antiangiogenic agents, such as sorafenib, also normalize tumor vasculature and thereby enhance the delivery of oxygen to the core of the tumor. ${ }^{16,26,27}$ The duration of increased tumor oxygenation, which can enhance the tumoricidal effect of radiation, is however believed to be temporary. Consequently, radiotherapy given during this normalization window might boost the synergistic effect of combined therapy. ${ }^{28}$ The optimal interval and sequence 


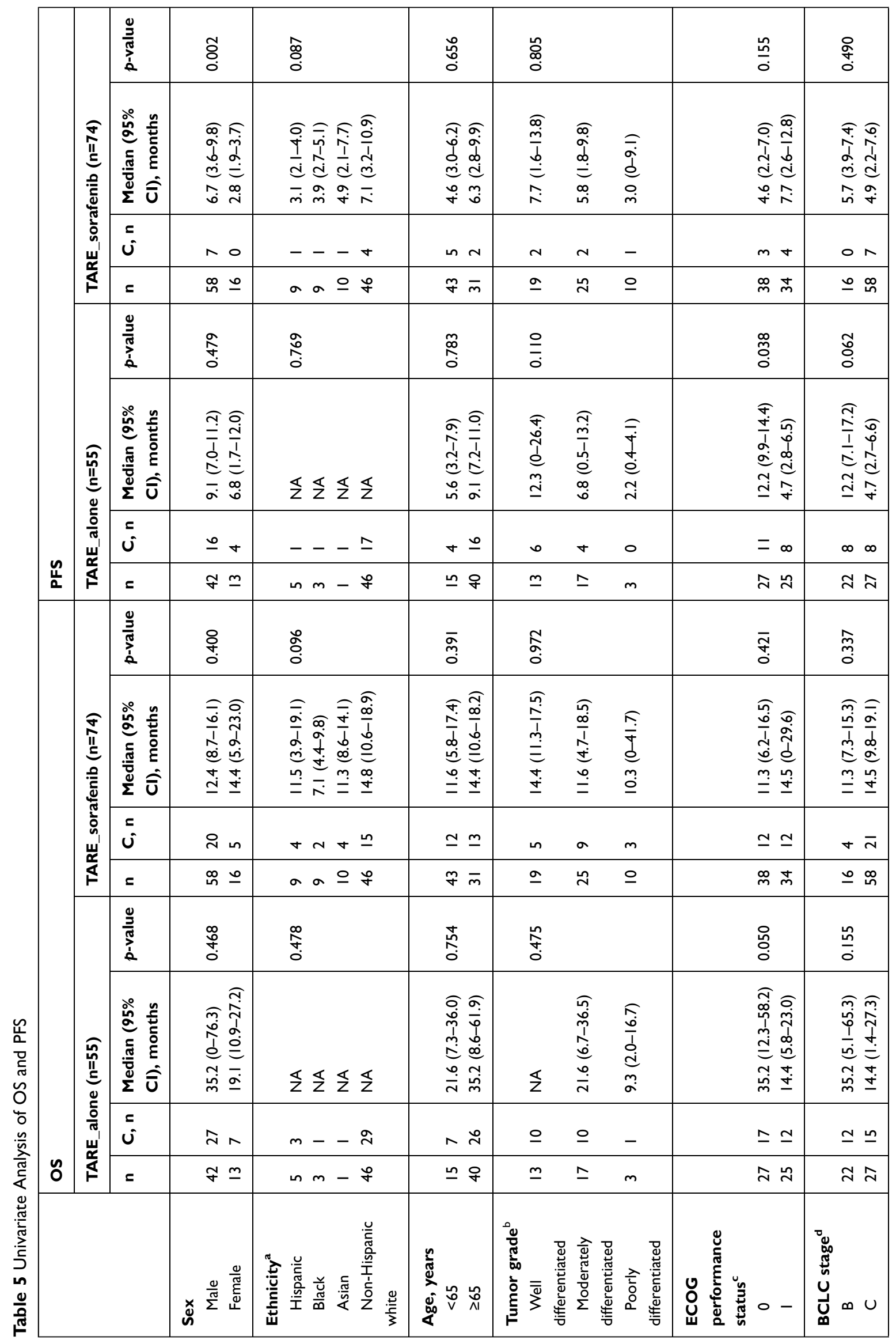




\begin{tabular}{|c|c|c|c|c|c|c|c|c|c|}
\hline$\underset{\substack{\infty \\
0}}{\stackrel{\infty}{0}}$ & $\frac{\bar{\infty}}{0}$ & $\stackrel{\stackrel{\sim}{+}}{\stackrel{0}{0}}$ & & $\frac{\text { సิ }}{0}$ & 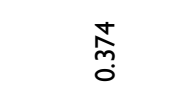 & 음 & $\begin{array}{l}\text { ֶू } \\
\text { Oे }\end{array}$ & & $\begin{array}{l}\bar{\circ} \\
\circ\end{array}$ \\
\hline 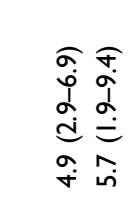 & 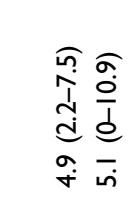 & 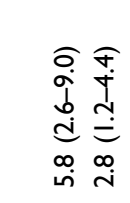 & & 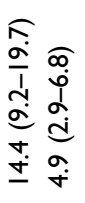 & 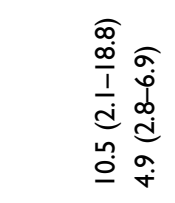 & 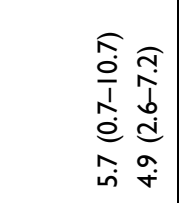 & 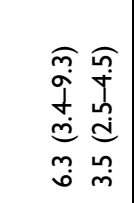 & & 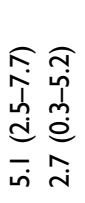 \\
\hline N n & $+m$ & $\ln N$ & & $m+$ & $\ln N$ & $\nabla m$ & $n \sim$ & & $\wedge 0$ \\
\hline$\hat{\jmath}$ & 必 & 능 & & a & 유 亡ั & 하 우 & $\hat{千} \curvearrowright$ & & $\bar{r} m$ \\
\hline$\frac{\stackrel{\infty}{=}}{0}$ & I & I & & 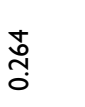 & $\begin{array}{l}\infty \\
\mathscr{O} \\
O\end{array}$ & I & ¿̊̊ & & $\frac{\underline{n}}{0}$ \\
\hline 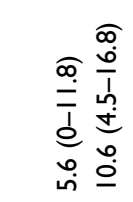 & 11 & 11 & & 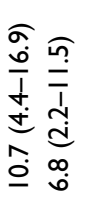 & 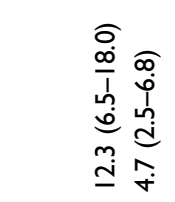 & 11 & 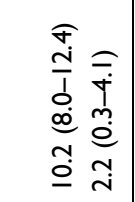 & & 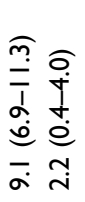 \\
\hline$-\underline{a}$ & 11 & 11 & & 드느 & $\underline{\underline{ }}$ ㅇ & 11 & $\stackrel{\infty}{\sim}$ & & $\underline{a}-$ \\
\hline$\underline{m} \underset{f}{ }$ & 11 & 11 & & 으 ํㅜㅁ & 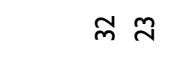 & 11 & $\stackrel{ }{\forall}$ 으 & & 윰 in \\
\hline 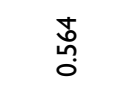 & 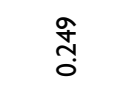 & $\frac{\infty}{0}$ & & $\begin{array}{l}\text { Lొ } \\
\infty \\
0 \\
0\end{array}$ & $\begin{array}{l}\stackrel{8}{0} \\
0\end{array}$ & 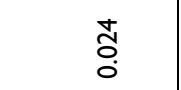 & סे & & $\begin{array}{l}\text { 今े } \\
\text { ०0 } \\
0\end{array}$ \\
\hline 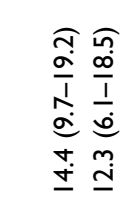 & 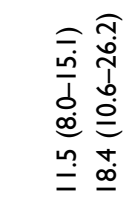 & 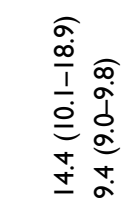 & & 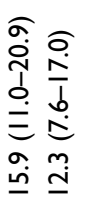 & 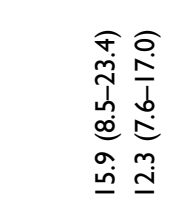 & 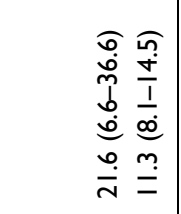 & 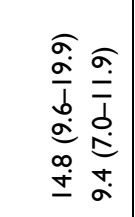 & & 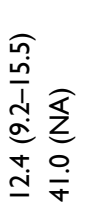 \\
\hline$\infty \simeq$ & $\pm=$ & $\bar{N}+$ & & $+\bar{\lambda}$ & 으 느 & $\simeq a$ & $\simeq \infty$ & & $\stackrel{ \pm}{d}-$ \\
\hline$\hat{\jmath}$ & 必 & 능 $\sigma$ & & a ถู & 유 亡ั & 하 우 & $\hat{f} \widehat{N}$ & & $\bar{r} m$ \\
\hline $\begin{array}{l}\text { L̊ } \\
\text { o. } \\
0\end{array}$ & I & I & & 岕 & $\stackrel{\text { ஸ̦ }}{0}$ & I & $\begin{array}{l}\overline{8} \\
\dot{0} \\
\mathrm{v}\end{array}$ & & 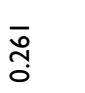 \\
\hline 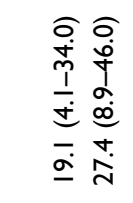 & 11 & 11 & & 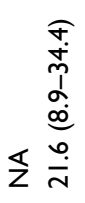 & 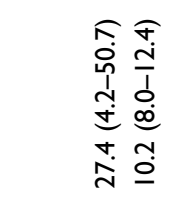 & 11 & 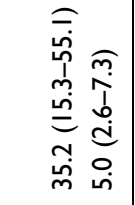 & & 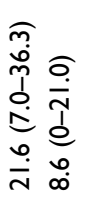 \\
\hline $0 \curvearrowright$ & 11 & 11 & & $\infty \stackrel{\llcorner}{\sim}$ & च 으 & 11 & సે + & & $\stackrel{m}{m}-$ \\
\hline$\underline{m} \mathcal{F}$ & 11 & 11 & & 으 무 & $\tilde{m} \tilde{\sim}$ & 11 & 눙 으 & & 온 nn \\
\hline 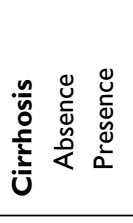 & 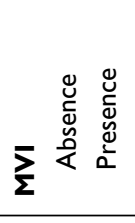 & 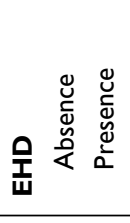 & 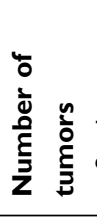 & $\frac{\frac{0}{0}}{\frac{0}{00}} \frac{\frac{0}{5}}{\Sigma}$ & 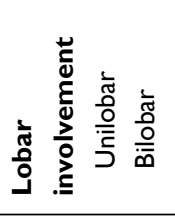 & 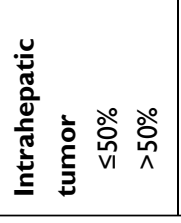 & 은 안 옷 & 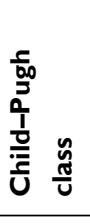 & $\ll \infty$ \\
\hline
\end{tabular}


Table 6 Multivariate Analysis of OS and PFS

\begin{tabular}{|c|c|c|c|c|c|c|c|c|}
\hline & \multicolumn{4}{|l|}{ OS } & \multicolumn{4}{|l|}{ PFS } \\
\hline & \multicolumn{2}{|c|}{ TARE_alone $(n=55)$} & \multicolumn{2}{|c|}{ TARE_sorafenib $(n=74)$} & \multicolumn{2}{|c|}{ TARE_alone $(n=55)$} & \multicolumn{2}{|c|}{ TARE_sorafenib $(\mathbf{N}=74)$} \\
\hline & HR (95\% Cl) & $p$-value & HR (95\% Cl) & p-value & HR (95\% Cl) & $p$-value & HR (95\% Cl) & $p$-value \\
\hline $\begin{array}{l}\text { Sex } \\
\text { Male } \\
\text { Female }\end{array}$ & - & - & - & - & - & $\begin{array}{l}- \\
-\end{array}$ & $\begin{array}{l}0.46(0.24-0.87) \\
1.00\end{array}$ & 0.018 \\
\hline $\begin{array}{l}\text { Ethnicity } \\
\text { Hispanic } \\
\text { Black } \\
\text { Asian } \\
\text { Non-Hispanic } \\
\text { white }\end{array}$ & $\begin{array}{l}- \\
- \\
-\end{array}$ & $\begin{array}{l}- \\
- \\
-\end{array}$ & $\begin{array}{l}2.22(0.82-5.89) \\
5.82(2.23-15.23) \\
1.33(0.55-3.23) \\
1.00\end{array}$ & $\begin{array}{l}0.118 \\
<0.001 \\
0.533\end{array}$ & $\begin{array}{l}- \\
- \\
-\end{array}$ & $\begin{array}{l}- \\
- \\
-\end{array}$ & $\begin{array}{l}1.66(0.76-3.6 I) \\
2.27(0.86-6.02) \\
2.29(1.05-4.98) \\
1.00\end{array}$ & $\begin{array}{l}0.208 \\
0.099 \\
0.037\end{array}$ \\
\hline $\begin{array}{c}\mathbf{E C O G}^{\mathbf{a}} \\
0 \\
1\end{array}$ & $\begin{array}{l}0.48(0.19-1.23) \\
1.00\end{array}$ & 0.125 & - & - & $\begin{array}{l}0.52(0.25-1.09) \\
1.00\end{array}$ & 0.084 & - & - \\
\hline $\begin{array}{l}\text { Lobar } \\
\text { involvement } \\
\text { Unilobar } \\
\text { Bilobar }\end{array}$ & $\begin{array}{l}0.5 I(0.19-1.32) \\
1.00\end{array}$ & 0.163 & - & - & $\begin{array}{l}0.43(0.20-0.92) \\
1.00\end{array}$ & 0.029 & - & - \\
\hline $\begin{array}{l}\text { Intrahepatic } \\
\text { tumor } \\
\quad \leq 50 \% \\
>50 \%\end{array}$ & - & $\begin{array}{l}- \\
-\end{array}$ & $\begin{array}{l}0.39(0.20-0.74) \\
1.00\end{array}$ & 0.004 & - & - & - & - \\
\hline $\begin{array}{l}\text { AFP, } \mathbf{n g} / \mathbf{m L} \\
\quad<400 \\
\geq 400\end{array}$ & $\begin{array}{l}0.27(0.09-0.8 I) \\
1.00\end{array}$ & 0.020 & $\begin{array}{l}0.50(0.27-0.93) \\
1.00\end{array}$ & 0.027 & $\begin{array}{l}0.49(0.20-1.22) \\
1.00\end{array}$ & 0.127 & $\begin{array}{l}0.52(0.3-0.88) \\
1.00\end{array}$ & 0.015 \\
\hline $\begin{array}{l}\text { Child-Pugh class } \\
\text { A } \\
\text { B }\end{array}$ & - & - & - & - & - & - & $\begin{array}{l}0.64(0.16-2.63) \\
1.00\end{array}$ & 0.535 \\
\hline
\end{tabular}

Notes: ${ }^{2}$ TARE_alone excluded ECOG $2(n=2)$ and ECOG $3(n=1)$; TARE_sorafenib excluded ECOG $2(n=2)$.

between sorafenib and TARE to achieve an adequate modification effect and optimal dosage of sorafenib when used in combination with TARE are currently unsettled. Intervals between sorafenib and TARE in published works have varied considerably. ${ }^{14,16}$ In the latest prospective randomized controlled trial in advanced-HCC patients comparing survival outcome between sorafenib alone and combination of sorafenib and TARE, initiation of sorafenib within 3 days after TARE did not provide additional survival benefit (OS 11.4 vs 12.1 months, HR 1.01, 95\% CI $0.81-1.25 ; p=0.9529) .{ }^{29}$ Interestingly, a majority of our patients $(90.5 \%)$ had been treated with sorafenib before TARE, and over half (58.1\%) had also received reduced dosages of sorafenib, but many still realized some benefits. On the basis of these observations, we hypothesized that initiation of sorafenib for a period prior to sorafenib could possibly improve survival outcomes, even if sorafenib is not given at full dosage.

Prior studies have reported that low serum AFP, unilobar involvement, IHT $<25 \%$, no EHD, no MVI, BCLC stage B, and Child-Pugh A were associated with better survival outcomes in patients receiving TARE. ${ }^{10,12,13}$ In this work, multivariate analysis revealed AFP as a prognostic factor for OS in both the TARE_alone $(H R$ 0.27, $p=0.02)$ and TARE_sorafenib (HR $0.5, p=0.027$ ) subgroups. TARE_alone patients with AFP $<400 \mathrm{ng} / \mathrm{mL}$ also had unexpectedly longer median OS than those with AFP $\geq 400 \mathrm{ng} / \mathrm{mL}$ (35.2 vs 5.0 months, $p<0.001$ ). These findings suggest that our treatment scheme is especially effective for patients with AFP $<400 \mathrm{ng} / \mathrm{mL}$. IHT was the other clinically meaningful prognostic factor for $\mathrm{OS}$ in the 

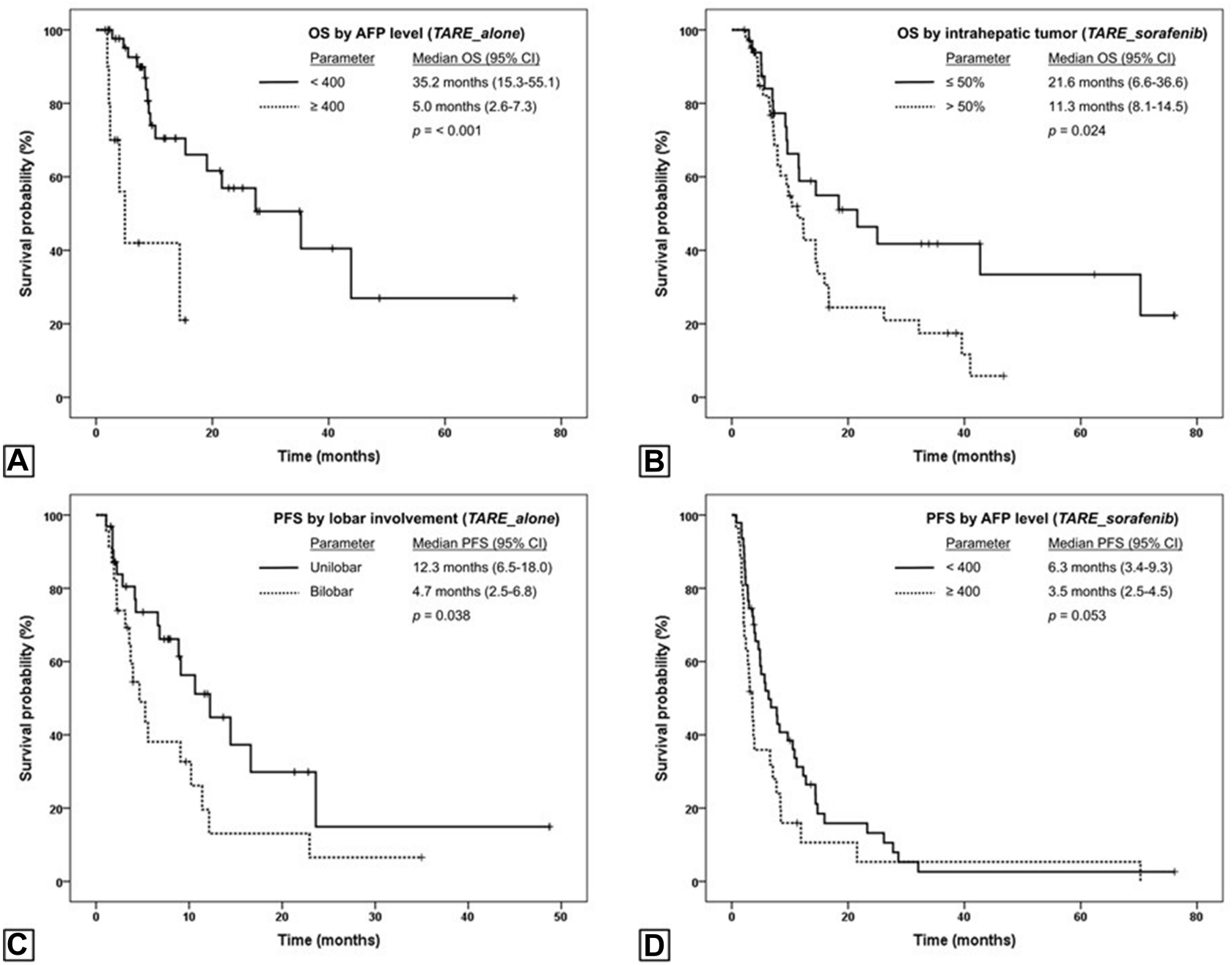

Figure I Kaplan-Meier curves stratified by prognostic factors showing, significantly longer OS durations for the (A) TARE_alone subgroup with AFP <400 ng/mL and (B) TARE_sorafenib subgroup with intrahepatic tumor $\leq 50 \%$, and longer median PFS durations for the (C) TARE_alone subgroup with unilobar involvement and (D) TARE_sorafenib subgroup with AFP $<400 \mathrm{ng} / \mathrm{mL}$.

TARE_sorafenib subgroup (HR 0.39, $p=0.004)$, supporting its use as a powerful differentiator along with ADFs as done in this work.

Multivariate analysis on the TARE_alone subgroup showed lobar involvement to be the only prognostic factor for PFS (HR 0.43, $p=0.029$ ). Given that lobar involvement was associated not only with DB but also with technical and dosimetric aspects of TARE, the markedly different PFS observed between unilobar and bilobar involvement (12.3 vs 4.7 months, $p=0.038$ ) was not unreasonable. AFP was once

Table 7 Incidence of AEs by Treatment

\begin{tabular}{|l|l|l|l|}
\hline & TARE_alone & TARE_sorafenib & TARE_alternative \\
\hline $\begin{array}{l}\text { Number of procedures } \\
\text { Number of procedures with AEs (\%) }\end{array}$ & 66 & 79 & 30 \\
\hline $\begin{array}{l}\text { Number of AEs } \\
\text { All }\end{array}$ & $162(80.3)$ & $73(92.4)$ & $23(76.6)$ \\
Grade 3-4 (\%) & $24(14.8)$ & 300 & 87 \\
\hline $\begin{array}{l}\text { Median AEs (range) } \\
\text { All }\end{array}$ & $2(0-10)$ & $21(7.0)$ & $6(6.9)$ \\
\hline Grade 3-4 & $2(1-6)$ & $3(0-11)$ & $3(0-8)$ \\
\hline
\end{tabular}


Table 8 Pertinent AEs by Treatment

\begin{tabular}{|c|c|c|c|c|c|c|}
\hline \multirow[t]{2}{*}{ Pertinent AEs } & \multicolumn{2}{|c|}{ TARE_alone } & \multicolumn{2}{|c|}{ TARE_sorafenib } & \multicolumn{2}{|c|}{ TARE_alternative } \\
\hline & Grade I-2 & Grade 3-4 & Grade I-2 & Grade 3-4 & Grade I-2 & Grade 3-4 \\
\hline \multicolumn{7}{|l|}{ Constitutional symptoms } \\
\hline Fever & $4(6.1)$ & 0 & $2(2.5)$ & 0 & I (3.2) & 0 \\
\hline Fatigue & $33(50.0)$ & 0 & $44(55.7)$ & 0 & $13(41.9)$ & 0 \\
\hline Weight loss & $2(3.0)$ & 0 & $3(3.8)$ & 0 & I (3.2) & 0 \\
\hline Loss of appetite & $3(4.5)$ & 0 & $9(11.4)$ & 0 & $2(6.5)$ & 0 \\
\hline Dermatological & I (I.5) & 0 & $19(24.1)$ & $2(2.5)$ & I (3.2) & 0 \\
\hline \multicolumn{7}{|l|}{ Gastrointestinal disorders } \\
\hline Diarrhea & 0 & 0 & $15(19.0)$ & 0 & 0 & 0 \\
\hline Nausea or vomiting & $14(2 \mid .2)$ & 0 & $34(43.0)$ & 0 & $9(29.1)$ & 0 \\
\hline Abdominal discomfort & $10(15.1)$ & $3(4.5)$ & $29(36.7)$ & $\mathrm{I}(\mathrm{l} .3)$ & II (35.5) & 0 \\
\hline Anorexia & $3(4.5)$ & 0 & $6(7.6)$ & 0 & I (3.2) & 0 \\
\hline Constipation & $2(3.0)$ & 0 & $5(6.3)$ & 0 & 0 & 0 \\
\hline Gastroesophageal varices hemorrhage & 0 & 0 & 0 & $2(2.5)$ & 0 & I (3.2) \\
\hline Gastritis & 0 & 0 & $\mathrm{I}(\mathrm{l} .3)$ & 0 & 0 & $\mathrm{I}(3.2)$ \\
\hline Pancreatitis & 0 & I ( $(1.5)$ & 0 & 0 & 0 & 0 \\
\hline Cholecystitis & 0 & 0 & 0 & 0 & 0 & I (3.2) \\
\hline Intra-abdominal bleeding & 0 & I (I.5) & 0 & $\mathrm{I}(\mathrm{l} .3)$ & 0 & 0 \\
\hline \multicolumn{7}{|l|}{ Liver disorders } \\
\hline Ascites & $4(6.1)$ & $4(6.1)$ & $6(7.6)$ & $2(2.5)$ & $4(12.9)$ & $2(6.5)$ \\
\hline REILD & 0 & 0 & 0 & 0 & 0 & 0 \\
\hline Hepatic encephalopathy & 0 & $3(4.5)$ & 0 & $2(2.5)$ & 0 & 0 \\
\hline \multicolumn{7}{|l|}{ Respiratory symptoms } \\
\hline Dyspnea & $4(6.1)$ & I (I.5) & $4(5.1)$ & 0 & $3(9.7)$ & 0 \\
\hline Cough & $\mathrm{I}(\mathrm{l} .5)$ & 0 & 0 & 0 & 0 & 0 \\
\hline Pleural effusion & 0 & 0 & 0 & $\mathrm{I}(1.3)$ & & \\
\hline Radiation-induced pneumonitis & 0 & 0 & 0 & 0 & 0 & 0 \\
\hline \multicolumn{7}{|l|}{ Laboratory abnormalities } \\
\hline Hyperbilirubinemia & $13(19.7)$ & $3(4.5)$ & $24(30.4)$ & $\mathrm{I}(\mathrm{I} .3)$ & $7(22.6)$ & 0 \\
\hline Hypoalbuminemia & $10(15.1)$ & 0 & $19(24.1)$ & 0 & $6(19.3)$ & 0 \\
\hline Anemia & $5(7.5)$ & 0 & II (13.9) & 0 & $6(19.3)$ & I (3.2) \\
\hline Thrombocytopenia & $12(18.2)$ & 0 & $17(2 \mid .5)$ & $3(3.8)$ & $10(32.3)$ & 0 \\
\hline Leukopenia & II (16.7) & $2(3.0)$ & $13(16.4)$ & $3(3.8)$ & $5(16.1)$ & 0 \\
\hline Renal dysfunction & 0 & 0 & $2(2.5)$ & 0 & 0 & 0 \\
\hline Increased AST & $4(6.0)$ & 0 & $8(10.1)$ & 0 & $4(12.9)$ & 0 \\
\hline Increased ALT & $2(3.0)$ & 0 & $2(2.5)$ & $\mathrm{I}(\mathrm{I} .3)$ & $3(9.7)$ & 0 \\
\hline Increased ALP & $\mathrm{I}(\mathrm{l} .5)$ & 0 & $7(8.9)$ & 0 & $2(6.5)$ & 0 \\
\hline \multicolumn{7}{|l|}{ Other } \\
\hline Electrolyte imbalance & 0 & $\mathrm{I}(\mathrm{l} .5)$ & $2(2.5)$ & 0 & 0 & 0 \\
\hline Sepsis & 0 & I (I.5) & 0 & $2(2.5)$ & 0 & 0 \\
\hline
\end{tabular}

Abbreviations: AST, aspartate aminotransferase; ALT, alanine aminotransferase; ALP, alkaline phosphatase.

implicated on multivariate analysis as a statistically significant parameter for PFS in the TARE_sorafenib subgroup, along with sex. Asian etnicity was not considered reliable, because of the small sample.

All our treatment regimens should be considered safe, as evidenced by only $9.3 \%$ grade $3-4$ AEs and no treatment-related deaths (grade 5), radiation pneumonitis, or REILD. The most common AEs - fatigue, nausea/ vomiting, abdominal discomfort, hyperbilirubinemia, and thrombocytopenia - were consistent with the results of existing studies. ${ }^{8-10,12,13}$ Although AEs were more common in the TARE_sorafenib subgroup, $>90 \%$ were grade 
1-2. Incidence of sorafenib-related AEs in our study was actually lower than in several prospective studies. ${ }^{22,30}$ This may be because in $55.7 \%$ of TARE_sorafenib procedures, the dosage of sorafenib was reduced to alleviate sorafenibrelated symptoms. In addition, most of these patients had been on a continued sorafenib regimen for a period prior to TARE, and thus were known to tolerate sorafenib at the time of TARE. The severity of some AEs in our study might have been overestimated, because patients with cirrhosis or portal hypertension may have had abnormal or marginally abnormal baseline characteristics (eg, hyperbilirubinemia, elevated liver enzymes, or thrombocytopenia), which are easily exacerbated by treatment. Furthermore, our follow-up was not limited to 3 or 6 months, but lasted until the patient's death, last followup, or change in treatment. The results during the lengthy follow-up could have been confounded by disease progression or underlying liver impairment. Therefore, it was not surprising to see a higher incidence of grade 3-4 AEs, particularly hepatic-related symptoms, in our TARE_alone patients, who had much longer OS and PFS than the others.

Our study has four major strengths. First, our findings are more generalized than those from clinical trials, as our study was conducted using real-world patient data from a large routine practice, where patients have a variety of DBs and disease characteristics and undergo various treatments. Second, stratification by DB and treatment has led to more specific results for each subgroup. Third, the assessment of critical features, such as IHT and ADFs, is practical and valid for treatment stratification. IHT $\leq 50 \%$ or $>50 \%$ are relatively coarse assessments allowing multidisciplinary team members to do this easily. Each descriptor of ADFs (eg, infiltrative HCC, MVI, and EHD), irrespective of whether patients present with them alone or together, resulted in the same treatment recommendation of TARE_sorafenib. Pooling all these features into a single group, namely ADFs, makes an algorithm easy to use. Finally, our study included patients treated with TARE and sorafenib, whose outcomes have not been investigated extensively, and we successfully revealed survival benefits of this combined treatment in a specific subgroup: IHD $\leq 50 \%$ without ADFs. The limitations of this study mostly relate to its retrospective nature. The number of samples in some subgroups was too low or too heterogeneous to be meaningful. Besides, uncontrollable factors, such as delayed treatment because of insurance issues or suboptimal treatment resulting from patient noncompliance, may have affected treatment outcomes.

On the basis of the findings stemming from this work, we suggest additional studies for further exploration. First, AFP level seems to be one of the strongest independent prognostic factors for survival. Incorporating AFP level into treatment considerations, along with other DB parameters, could make the treatment algorithm more efficient. Second, the optimal interval and dosage of sorafenib when used in combination with TARE have not been fully established; therefore, further studies to determine these parameters might be the key to successful combined therapy. Third, assessing the failure patterns of any given treatment might reveal pitfalls that can be pathways for improvement. Finally, the most recent prospective trial comparing survival outcomes between the combination of atezolizumab and bevacizumab versus sorafenib in unresectable HCC showed better OS and PFS in the dualtherapy arm, with HR $0.58 .{ }^{31}$ Looking ahead, atezolizumab and bevacizumab in combination with TARE could be explored as an alternative treatment option, especially for patients with relatively high DB.

\section{Conclusion}

Our institutional treatment approach seems to be a promising strategy for unresectable HCC patients. TARE_alone could provide an impressive survival outcome in patients with IHT $\leq 50 \%$ without ADFs, and was particularly prominent in the setting of unilobar $\mathrm{HCC}$ and serum AFP $<400 \mathrm{ng} / \mathrm{mL}$. TARE_sorafenib resulted in some extra grade 1-2 AEs compared to TARE only, and thus should be preserved for patients with IHT $>50 \%$ or presence of ADFs. Patients with IHT $\leq 50 \%$ and presence of ADFs with serum AFP $<400 \mathrm{ng} / \mathrm{mL}$ are most likely to benefit from this combined treatment.

\section{Abbreviations}

OS, overall survival; PFS, progression-free survival; HCC, hepatocellular carcinoma; TARE, transarterial radioembolization; IHT, intrahepatic tumor; ADFs, advanced/aggressive disease features; $\mathrm{AE}$, adverse event; $\mathrm{DB}$, disease burden; BCLC, Barcelona Clinic Liver Cancer; TACE, transarterial chemoembolization; MVI, macroscopic vascular invasion; EHD, extrahepatic disease; CT, computed tomography; ${ }^{99 \mathrm{~m}} \mathrm{Tc}$ MAA, technetium $99 \mathrm{~m}$ macroaggregated albumin; ECOG, Eastern Cooperative Oncology Group; AFP, $\alpha$ fetoprotein; CTCAE, Common Terminology Criteria for Adverse Events; REILD, radioembolization-induced liver disease. 


\section{Ethical Approval}

Patient enrollment and all treatment procedures performed in this study were in accordance with the 1964 Declaration of Helsinki and later amendments and the ethical standards of human experimentation, consisting of the institutional review board and the Food and Drug Administration Humanitarian Device Exemption (FDA HDE) guidelines. This retrospective study was approved by the University of Texas MD Anderson Cancer Center Institutional Review Board.

\section{Informed Consent}

A waiver of informed consent was granted by our institutional review board for this retrospective study. Patient data used complied with all institutional data-protection and privacy regulations.

\section{Disclosure}

AM has received research grants from BTG International, Sirtex Medical, and ABK Medical and serves as a consultant for BTG, Sirtex, and Boston Scientific. JDK has received a research grant from Cynvenio Biosystems and is on a speaker bureau of Angiodynamics. He reports personal fees from BTG and Argon, and serves on an advisory board for Boston Scientific. BC serves on an advisory board for Advanced Accelerator Applications and Clovis Oncology. BCO. has received a research grant from Siemens Healthineers and serves as a consultant for Koo Foundation. SCK has received research grants from BTG International and GE Healthcare and serves as a consultant for BTG International, Terumo Medical, Sirtex Medical, and ABK Biomedical. The authors report no other conflicts of interest in this work.

\section{References}

1. Liver cancer [homepage on the Internet]. The global cancer observatory cancer fact sheets; 2018. Available from: http://gco.iarc.fr/today/data/ factsheets/cancers/11-Liver-fact-sheet.pdf. Accessed May 2, 2019.

2. Llovet JM, Beaugrand M. Hepatocellular carcinoma: present status and future prospects. $J$ Hepatol. 2003;38(Suppl 1):S136-149. doi:10. 1016/S0168-8278(02)00432-4

3. Tabrizian P, Roayaie S, Schwartz ME. Current management of hepatocellular carcinoma. World J Gastroenterol. 2014;20(30):1022 3-10237. doi:10.3748/wjg.v20.i30.10223

4. Forner A, Reig ME, de Lope CR, Bruix J. Current strategy for staging and treatment: the BCLC update and future prospects. Semin Liver Dis. 2010;30(1):61-74. doi:10.1055/s-0030-1247133

5. Heimbach JK, Kulik LM, Finn RS, et al. AASLD guidelines for the treatment of hepatocellular carcinoma. Hepatology. 2018;67(1): 358-380. doi: $10.1002 /$ hep. 29086
6. Bradford R, McKinney JM. Treatment planning part I: vascular considerations associated with safety and efficacy in radioembolization. In: Pasciak ASBY, McKinney JM, editors. Handbook of Radioembolization. Boca Raton, FL: CRC Press; 2017:53-64.

7. Srinivas SM, Shrikanthan S, Yu N, Kost SD, Gurajala R, Karuppasamy K. Treatment planning part II: procedure simulation and prognostication. In: Pasciak ASBY, McKinney JM, editors. Handbook of Radioembolization. Boca Raton, FL: CRC Press; 2017:65-86.

8. Hilgard P, Hamami M, Fouly AE, et al. Radioembolization with yttrium-90 glass microspheres in hepatocellular carcinoma: European experience on safety and long-term survival. Hepatology. 2010;52(5):1741-1749. doi:10.1002/hep.23944

9. Kulik LM, Carr BI, Mulcahy MF, et al. Safety and efficacy of $90 \mathrm{Y}$ radiotherapy for hepatocellular carcinoma with and without portal vein thrombosis. Hepatology. 2008;47(1):71-81. doi:10.1002/ hep. 21980

10. Mazzaferro V, Sposito C, Bhoori S, et al. Yttrium-90 radioembolization for intermediate-advanced hepatocellular carcinoma: a Phase 2 study. Hepatology. 2013;57(5):1826-1837. doi:10.1002/hep.26014

11. Murthy R, Kamat P, Nunez R, Salem R. Radioembolization of yttrium-90 microspheres for hepatic malignancy. Semin Intervent Radiol. 2008;25(1):48-57. doi:10.1055/s-2008-1052306

12. Salem R, Lewandowski RJ, Mulcahy MF, et al. Radioembolization for hepatocellular carcinoma using Yttrium-90 microspheres: a comprehensive report of long-term outcomes. Gastroenterology. 2010;138(1):52-64. doi:10.1053/j.gastro.2009.09.006

13. Sangro B, Carpanese L, Cianni R, et al. Survival after yttrium-90 resin microsphere radioembolization of hepatocellular carcinoma across Barcelona clinic liver cancer stages: a European evaluation. Hepatology. 2011;54(3):868-878. doi:10.1002/hep.24451

14. Rana N, Ju AW, Bazylewicz M, et al. Yttrium-90 radioembolization in patients with hepatocellular carcinoma who have previously received sorafenib. Front Oncol. 2013;3:323. doi:10.3389/fonc.20 13.00323

15. Ricke J, Bulla K, Kolligs F, et al. Safety and toxicity of radioembolization plus sorafenib in advanced hepatocellular carcinoma: analysis of the European multicentre trial SORAMIC. Liver Int. 2015;35 (2):620-626. doi:10.1111/liv.12622

16. Salman A, Simoneau E, Hassanain M, et al. Combined sorafenib and yttrium-90 radioembolization for the treatment of advanced hepatocellular carcinoma. Curr Oncol. 2016;23(5):e472-e480. doi:10.3747/ co. 23.2827

17. Hepatocellular carcinoma [homepage on the Internet]. Cancer treatment algorithm; 2017. Available from: https://www.mdanderson.org/ content/dam/mdanderson/documents/for-physicians/algorithms/can cer-treatment/ca-treatment-hepatocellular-web-algorithm.pdf. Accessed September 15, 2018.

18. Lewandowski RJ, Salem R. Yttrium-90 radioembolization of hepatocellular carcinoma and metastatic disease to the liver. Semin Intervent Radiol. 2006;23(1):64-72. doi:10.1055/s-2006-939842

19. Oken MM, Creech RH, Tormey DC, et al. Toxicity and response criteria of the eastern cooperative oncology group. Am J Clin Oncol. 1982;5(6):649-655. doi:10.1097/00000421-198212000-00014

20. Johnson PJ, Berhane S, Kagebayashi C, et al. Assessment of liver function in patients with hepatocellular carcinoma: a new evidence-based approach-the ALBI grade. J Clin Oncol. 2015;33 (6):550-558. doi:10.1200/JCO.2014.57.9151

21. TheraSphere (Yttrium-90 Glass Microspheres) package insert. Boston Scientific, Minneapolis, MN, USA> Available from: https:// www.bostonscientific.com/en-US/products/cancer-therapies/thera sphere-y90-glass-microspheres/therasphere-y 90 -microspheres-briefsummary.html. Accessed August 12, 2020.

22. Llovet JM, Ricci S, Mazzaferro V, et al. Sorafenib in advanced hepatocellular carcinoma. N Engl J Med. 2008;359(4):378-390. doi:10.1056/NEJMoa0708857 
23. Common Terminology Criteria for Adverse Events (CTCAE) Version 5.0 [homepage on the Internet]. Common terminology criteria for adverse events; 2017. Available from: https://ctep.cancer.gov/proto coldevelopment/electronic_applications/docs/CTCAE_v5_Quick Reference_5x7.pdf. Accessed September 15, 2018.

24. Gil-Alzugaray B, Chopitea A, Inarrairaegui M, et al. Prognostic factors and prevention of radioembolization-induced liver disease. Hepatology. 2013;57(3):1078-1087. doi:10.1002/hep.26191

25. Eisenhauer EA, Therasse P, Bogaerts J, et al. New response evaluation criteria in solid tumours: revised RECIST guideline (version 1.1). Eur J Cancer. 2009;45(2):228-247. doi:10.1016/j.ejca.2008. 10.026

26. Senan S, Smit EF. Design of clinical trials of radiation combined with antiangiogenic therapy. Oncologist. 2007;12(4):465-477. doi:10.16 34/theoncologist.12-4-465

27. Cao Y, Liu Q. Therapeutic targets of multiple angiogenic factors for the treatment of cancer and metastasis. Adv Cancer Res. 2007; 97:203-224.
28. Winkler F, Kozin SV, Tong RT, et al. Kinetics of vascular normalization by VEGFR2 blockade governs brain tumor response to radiation: role of oxygenation, angiopoietin-1, and matrix metalloproteinases. Cancer Cell. 2004;6(6):553-563. doi:10.1016/j.ccr.2004.10.011

29. Ricke J, Klümpen HJ, Amthauer $\mathrm{H}$, et al. Impact of combined selective internal radiation therapy and sorafenib on survival in advanced hepatocellular carcinoma. J Hepatol. 2019;71(6):11641174. doi:10.1016/j.jhep.2019.08.006

30. Vilgrain V, Pereira H, Assenat E, et al. Efficacy and safety of selective internal radiotherapy with yttrium-90 resin microspheres compared with sorafenib in locally advanced and inoperable hepatocellular carcinoma (SARAH): an open-label randomised controlled Phase 3 trial. Lancet Oncol. 2017;18(12):1624-1636. doi:10.1016/ S1470-2045(17)30683-6

31. Finn RS, Qin S, Ikeda M, et al. Atezolizumab plus bevacizumab in unresectable hepatocellular carcinoma. $N$ Engl J Med. 2020;382 (20):1894-1905. doi:10.1056/NEJMoa1915745

\section{Publish your work in this journal}

The Journal of Hepatocellular Carcinoma is an international, peerreviewed, open access journal that offers a platform for the dissemination and study of clinical, translational and basic research findings in this rapidly developing field. Development in areas including, but not limited to, epidemiology, vaccination, hepatitis therapy, pathology and molecular tumor classification and prognostication are all considered for publication. The manuscript management system is completely online and includes a very quick and fair peer-review system, which is all easy to use. Visit http://www.dovepress.com/ testimonials.php to read real quotes from published authors. 